\title{
Team sport players individual acceleration-speed profile in-situ: a proof of concept in professional football.
}

Jean-Benoit Morina, Yann Le Mata, Cristian Osgnach ${ }^{b}$, Alessandro Pilatic, Pierre Samozino ${ }^{d}$, Pietro E di Prampero ${ }^{b, e}$

a Univ Lyon, UJM-Saint-Etienne, Laboratoire Interuniversitaire de Biologie de la Motricité, EA 7424, F-42023, Saint-Etienne, France.

${ }^{b}$ Department of Sport Science, Exelio SRL, Udine, Italy

c Performance Department, Genoa CFC, Genova, Italy

d Univ Savoie Mont Blanc, Laboratoire Interuniversitaire de Biologie de la Motricité, EA 7424, F-73000 Chambéry, France

e Emeritus Professor of Physiology, University of Udine, Italy

\section{Corresponding author:}

Pr Jean-Benoit Morin, LIBM

Campus Santé Innovations - Batiment IRMIS

10 rue de la Marandière - 42270 Saint-Priest-en-Jarez, FRANCE

Email: jean.benoit.morin@univ-st-etienne.frＴel:0033477421875Ｔwitter : @jb_morin

KEYWORDS: soccer; testing; running; sprint; GPS

\section{ABSTRACT}

Assessing football players' sprint mechanical outputs is key to the performance management process (e.g. talent identification, training, monitoring, return-to-sport). This is possible using linear sprint testing to derive force-velocitypower outputs (in laboratory or field settings), but (i) testing requires specific efforts and (ii) the movement assessed is not specific to the football playing tasks. This proof-of-concept short communication presents a method to derive the players' individual acceleration-speed (AS) profile in-situ, i.e. from global positioning system data collected over several football sessions (without running specific tests). Briefly, raw speed data collected in 16 professional male football players over several training sessions were plotted, and for each $0.2 \mathrm{~m} / \mathrm{s}$ increment in speed from $3 \mathrm{~m} / \mathrm{s}$ up to the individual top-speed reached, maximal acceleration output was retained to generate a linear AS profile. Results showed highly linear AS profiles for all players (all $\mathrm{R}^{2}>0.984$ ) which allowed to extrapolate the theoretical maximal speed and accelerations as the individual's sprint maximal capacities. Good reliability was observed between AS profiles determined 2 weeks apart for the players tested, and further research should focus on deepening our understanding of these methodological features. Despite the need for further explorations (e.g. comparison with conceptually close forcevelocity assessments that require, isolated and not football-specific linear sprint tests), this in-situ approach is promising and allows direct assessment of team sport players within their specific acceleration-speed tasks. This opens several perspectives in the performance and injury prevention fields, in football and other team sports, and the possibility to "test players without testing them".

All co-authors have significantly contributed to this work, reviewed and approved this pre-print.

Cite as: Morin J-B, Le Mat Y, Osgnach C, Pilati A, Samozino P, di Prampero PE. Team sport players individual accelerationspeed profile in-situ: a proof of concept in professional football. SportRxiv, 2020. 


\section{Introduction}

Sprinting is a key physical determinant of performance in team sports (Faude et al., 2012; Haugen et al., 2014). Thus, assessing and monitoring team sport players individual sprint acceleration- or force-velocity profile is important to improve the training and injury management process (e.g. Jiménez-Reyes et al., 2020). Gold standard methods for ground reaction force measurement during sprint acceleration require instrumented treadmills or track-embedded multiple force plate systems, which is inaccessible to most athletes. For this reason, a simple field method based on position-time data and Newtonian laws of motion applied to the athlete's center of mass has been recently presented (Morin et al., 2019; Samozino et al., 2016). Due to a good ratio between overall validity and the overall reliability and simplicity of the model inputs (i.e. mainly athlete's body mass and center of mass position or speed over time), this method has been used in both training and medical practice and research (e.g. Mendiguchia et al., 2014). However, it is based on a single linear test of acceleration effort (from null to maximal running speed) that requires preparation and organization, and that is not specific to team sports such as football or rugby. In these team sports, athletes produce large amounts of linear accelerations and sprints of various durations, and starting from various levels of initial speed, and sometimes after a change of direction. Knowing the real "in-situ" sprint macroscopic forcevelocity profile of team sport players could be of interest to (i) assess their acceleration sport-specific capabilities based on data collected "passively" (i.e. without specific intervention) during their actual practice, (ii) potentially save some testing time and physical/mental load induced by the aforementioned sprint testing protocol and (iii) bring continuous information to sport and medical staffs about players physical fitness without requiring specific testing and the associated reluctance. Assessing athletes' physical profile and capacity directly from real practice data (i.e. not isolated, laboratory-based or non-specific testing) has been done with race and training power output data "passive" collection in cycling, based on the "power record profile" concept (Pinot and Grappe, 2015, 2011) from which the method we propose here is inspired. In team sports, beyond the power output, the force-velocity profile informs on the acceleration capacities of the players at different velocities, which can be also characterized by the acceleration-speed (AS) profile. In other words, the AS profile represents the maximal forward acceleration capability of a player (resulting from propulsive force in the direction of running, according to Newtonian laws of motion) over the range of their running velocity spectrum. Conceptually, the information provided by the in-situ AS profile is close to the sprint forcevelocity profile explored during specific testing (linear sprint acceleration from zero to maximal running speed) (Morin et al., 2019; Samozino et al., 2016). The aim of this proof-of-concept study is to present a simple method to derive team sport players individual acceleration-speed profile from global positioning system (GPS) data collected over several training sessions.

\section{Method}

\subsection{Participants, procedures and equipment}

Sixteen male professional football players (mean \pm SD age of $25.3 \pm 3.8 \mathrm{yr}$; body mass $78.9 \pm 4.3 \mathrm{~kg}$; height $1.80 \pm 0.1 \mathrm{~m}$ ) gave their written informed consent to participate in this study. These football players were training and playing within the first team of an Italian first league professional club. Their training program during the data collection period (August 20, 2018 to September 23, 2018) included 5 football training sessions, 1 physical training gym session and 1 official football game per week. Official game data were not included in the analysis because the players did not carry their GPS units. In order to assess the reliability of 
the AS profile between collection periods, data were analyzed for comparison over two 2-week phases: from August 20 to September 2 (Phase1) and from September 10 to September 23 (Phase2).

During each football training session, players were constantly monitored with the same GPS unit (GPEXE Pro2, Exelio SRL, Italy, firmware version 0.13 ) inserted into tightly fit vests, between the upper sides of scapula blades. Position and speed data were collected at a sampling rate of $18 \mathrm{~Hz}$. The data of 13 players who completed at least 5 football training sessions ( $~ 90 \mathrm{~min}$ ) during both Phase1 and Phase2 were retained for further analyses. This minimal amount of 5 training sessions represent a cumulative total of $\sim 500.000$ raw data points, which allowed the collection of enough raw acceleration-speed data points to cover the 0 -tomaximal running speed range for each player.

Data collection for this retrospective analysis was performed within the typical training of the professional club, under usual technical and medical supervision. No specific intervention was therefore required for this study, which was performed according to Declaration of Helsinki.

\subsection{Data analysis}

The aim of the in-situ AS profile is to identify the linear relationship between a given running speed and the corresponding maximal acceleration generated in cumulated football practice data collected over a given period. More specifically, the GPS data collected over a defined time window yielded a cloud of accelerationspeed points (depending on the sampling frequency of the GPS devices used). A typical example of this scatter plot is presented in Fig. 1 (note that only positive acceleration values were displayed and retained for further analyses). This large set of raw data was first filtered with a custom Gaussian type procedure accounting for the time-averaged running acceleration. The individual AS profile was plotted based on the maximal acceleration the player could generate for every possible running speed over the measurement period, as follows. Within a running speed interval ranging between $3 \mathrm{~m} / \mathrm{s}$ and the individual maximal speed, the two maximal values of acceleration performed for each $0.2 \mathrm{~m} / \mathrm{s}$ subintervals (i.e. 3, 3.2, 3.4, $3.6 \mathrm{~m} / \mathrm{s}$ and $\mathrm{so}$ on) were selected for further analysis. This $3 \mathrm{~m} / \mathrm{s}$ threshold was chosen since maximal values of accelerations were rarely observed below this point (see Fig. 1), which is consistent with the fact that even at the very first steps of a standing or starting-block start, the center of mass velocity raises quickly above $3 \mathrm{~m} / \mathrm{s}$ within the first step (e.g. Morin et al., 2019; Nagahara et al., 2014). A first linear regression was fitted to these speed-acceleration points ( 70 data points depending on the individuals). Then, after fitting, the residuals were analyzed and outlier points were removed, in order to improve the linear regression fitting and the overall accuracy of the model variables. For each individual and phase tested, this procedure eventually provided $\sim 50$ data points (52 \pm 5 , range 43-63) from which the AS profile was derived using the updated linear regression model (for description of the linear modeling of sprint force-velocity profile, see (Morin et al., 2019; Rabita et al., 2015; Samozino et al., 2016)).

Finally, three main variables were derived to characterize the players AS profile and potentially be the variables of interest for further studies: $A_{0}$ is the theoretical maximal acceleration (y-intercept of the AS linear relationship); $S_{0}$ is the theoretical maximal running speed (x-intercept of the $A S$ relationship); $A S_{\text {slope }}$ is the slope, i.e. overall orientation of the AS profile (computed as $A S_{\text {slope }}=-A_{0} / S_{0}$ ).

\subsection{Statistics}

All data are presented as mean \pm standard deviation (SD). The quality of the linear fitting of the $A S$ relationships was assessed with $R^{2}$ values. The inter-Phase reliability for each variable was quantified through 
the change in the mean (systematic error) and the standard error of measurement (SEM, random error), both expressed in raw units and in percentage of mean values, between Phase1 and Phase2 data (Hopkins, 2000).

\section{Results}

All acceleration-speed individual profiles showed nearly perfect linear trends (all $R^{2}>0.984$, typical example in Fig. 1).

Figure 1. Typical example of an individual acceleration-speed profile obtained from the data of 8 training sessions spanned over 2 consecutive weeks in a professional football player. From the total $>700.000$ raw points, 51 points were selected (see methods) to compute the acceleration-speed linear profile. The dashed line shows the linear profile, from which theoretical maximal acceleration $\left(A_{0}=7.88 \mathrm{~m} / \mathrm{s}^{2}\right.$ in this example) and speed $\left(S_{0}=9.19 \mathrm{~m} / \mathrm{s}\right)$ were determined. Raw data below the $3 \mathrm{~m} / \mathrm{s} \mathrm{speed} \mathrm{threshold} \mathrm{were} \mathrm{partially} \mathrm{masked}$ for clarity.

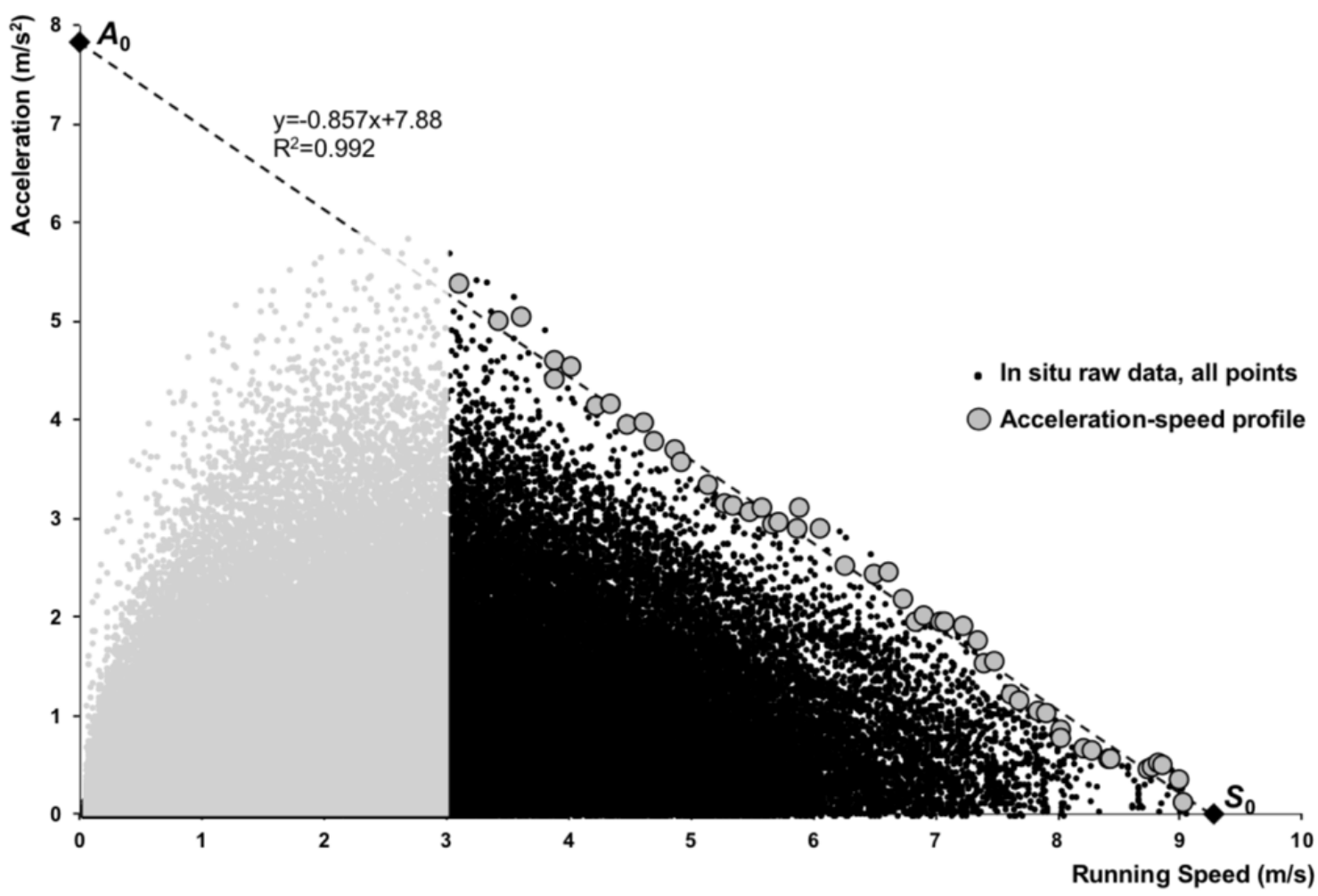

The main variables computed and between-Phase reliability are presented in Table 1.

Table 1. Main variables of the individual acceleration-speed profile for the two training phases analyzed.

\begin{tabular}{lccccc} 
& Phase1 & Phase2 & $\begin{array}{c}\text { Raw difference } \\
\text { (Phase2 - } \\
\text { Phase1) }\end{array}$ & $\begin{array}{c}\text { Raw difference } \\
\text { (\% from Phase1) }\end{array}$ & $\begin{array}{c}\text { Standard Error } \\
\text { of Measurement of Measurement } \\
\text { (raw units) }\end{array}$ \\
\hline $\boldsymbol{S}_{0}(\mathrm{~m} / \mathrm{s})$ & $9.21 \pm 0.43(8.41 ; 9.92)$ & $9.47 \pm 0.52(8.68 ; 10.3)$ & $0.26 \pm 0.43$ & 2.86 & 0.30 \\
$\boldsymbol{A}_{0}\left(\mathrm{~m} / \mathrm{s}^{2}\right)$ & $7.70 \pm 0.53(6.55 ; 8.43)$ & $7.20 \pm 0.40(6.72 ; 7.84)$ & $-0.50 \pm 0.59$ & -6.43 & 3.31 \\
AS slope $(1 / \mathrm{s})$ & $-0.839 \pm 0.086(-0.976 ;-0.660)$ & $-0.764 \pm 0.070(-0.976 ;-0.660)$ & $0.08 \pm 0.09$ & -9.03 & 0.41 \\
\hline
\end{tabular}

\section{Discussion}

The main result of this study is that an almost perfectly linear acceleration-speed relationship was observed for all the professional football players, which confirms that an in-situ individual AS profile may be computed from football GPS data passively collected and processed as described above. The range of $A_{0}$ and $S_{0}$ show that 
even in a highly trained population, who followed the same football training sessions, major inter-player differences were observed in acceleration-speed capacities.

This in-situ AS concept, which is easy to implement (no specific testing required, only GPS data passively collected throughout several training sessions), might lead to more specific and advanced assessment and monitoring of team sport players sprinting mechanical outputs. The potential applications are numerous, within the training, talent identification or injury management fields, as observed in recent studies using the concept of sprint acceleration force-velocity profiling (e.g. Haugen et al., 2020b; Jiménez-Reyes et al., 2018, 2020; Mendiguchia et al., 2014, 2016).

The reliability observed between Phase 1 and Phase2 (random error $<8 \%$, Table 1 ) is very good and in line with reliability observed during standardized sprint testing (Haugen et al., 2020a; Samozino et al., 2016). Note that, unexpectedly, the systematic differences in the individual AS profile between phases 1 and 2 is not negligible. These differences require further investigations and may be partly due to the reproducibility of the method and procedures proposed, the devices used (although similar units were used by each player), but also the actual changes in maximal running acceleration and speed induced by the several weeks of football training and games that occurred between Phase1 and Phase2. Indeed, recent research has shown that sprint acceleration force and velocity outputs changed over the course of a season in professional football players (Jiménez-Reyes et al., 2020). These inter-Phase systematic differences obtained here could also have been influenced by actual changes in players AS profile and/or changes in coaching instructions and training content that may have differed (e.g. more maximal speed-oriented stimuli) between Phase1 and Phase2.

Although direct comparison with existing literature is not possible, there is a conceptual proximity between the in-situ AS profile presented here and the force-velocity profile obtained during single, isolated, linear sprint tests. Indeed, $S_{0}$ is the same mechanical concept as " $V_{0}$ ", and by Newtonian laws of motion, $A_{0}$ and $F_{0}$ are two expressions of the same capacity (maximal acceleration in the forward direction of motion expressed in $\mathrm{m} / \mathrm{s}^{2}$ or the corresponding ground reaction force antero-posterior component per unit body mass expressed in $\mathrm{N} / \mathrm{kg}$ ). The typical values found in this study are remarkably close to those previously reported by different research groups in professional male football players: $S_{0}$ of $9-10 \mathrm{~m} / \mathrm{s}$ in the present study versus $V_{0}$ of 9.3 on average for (Jiménez-Reyes et al., 2020), 9.25 for (Jiménez-Reyes et al., 2018), or 9.2 for (Haugen et al., $2020 \mathrm{~b}, 2019$ ); $A_{0}$ of $7.2-7.7 \mathrm{~m} / \mathrm{s}^{2}$ in the present study versus $F_{0}$ of $7.11 \mathrm{~N} / \mathrm{kg}$ on average for (Jiménez-Reyes et al., 2020), 7.35 for (Jiménez-Reyes et al., 2018), or 8.4-8.5 for (Haugen et al., 2020b, 2019).

One limitation of this work is that official game data could not be pooled with training GPS data due to different systems used, thus questioning the extent to which individual AS profiles would differ by adding official game data. In addition, it is important to note that the quality of the linear fitting used to determine the AS profile highly depends on the quality of the sampled GPS signal, which depends on environmental, hardware and software characteristics, and may influence the application of the proposed method to different contexts. This is a track for future research on the topic, that could include (i) a comparison with "classical" isolated sprint testing AS or force-velocity profile, or other types of football players' effort assessment, (ii) the changes in AS profile over a full season or before-after injury, (iii) the effects of tactical coaching choices and associated training content orientation (e.g. small-sided games, acceleration or speed-oriented training, tactical periodization), (iv) the effects of strength and conditioning blocks (e.g. do the effects of heavy resistance training on $F_{0}$ (Morin et al., 2017) transfer to football-specific $A_{0}$ ?). 
More broadly, the main topic that should be addressed is the direct comparison of linear test force-velocity outcomes with those derived from the in-situ method proposed here, and the extent to which these pieces of information are interchangeable. Also, in order to narrow the time-focus down, research should clarify the minimal amount of data (i.e. training sessions and days/weeks of data collection) needed to provide reliable AS profiles. Finally, future works should test the possible use of this approach in other team sports, provided accurate individual acceleration and speed data are collected during practice.

In conclusion, keeping in mind the limitations and need for future works, the in-situ acceleration-speed profiling presented here allows to individually characterize the limits of team sport players acceleration and running speed capacities, in real football context (with or without ball, opponents, teammates, accelerations in different directions), from football practice GPS data: "testing the players without testing them".

\section{Conflict of interest statement}

Authors Cristian Osgnach and Pietro E di Prampero are scientific consultants for Exelio SRL, the company which provided the GPS units used in this study.

Other authors have no conflict of interest to declare.

\section{References}

Faude, 0., Koch, T., Meyer, T., 2012. Straight sprinting is the most frequent action in goal situations in professional football. J. Sports Sci. 30, 625-631. https://doi.org/10.1080/02640414.2012.665940

Haugen, T., Tønnessen, E., Hisdal, J., Seiler, S., 2014. The role and development of sprinting speed in soccer. Int. J. Sports Physiol. Perform. 9, 432-41. https://doi.org/10.1123/ijspp.2013-0121

Haugen, T.A., Breitschädel, F., Samozino, P., 2020a. Power-Force-Velocity Profiling of Sprinting Athletes: Methodological and Practical Considerations When Using Timing Gates. J. strength Cond. Res. 34, 1769-1773. https://doi.org/10.1519/JSC.0000000000002890

Haugen, T.A., Breitschädel, F., Seiler, S., 2020b. Sprint mechanical properties in soccer players according to playing standard, position, age and sex. J. Sports Sci. 38, 1070-1076.

https://doi.org/10.1080/02640414.2020.1741955

Haugen, T.A., Breitschädel, F., Seiler, S., 2019. Sprint mechanical variables in elite athletes: Are forcevelocity profiles sport specific or individual? PLoS One 14, e0215551.

https://doi.org/10.1371/journal.pone.0215551

Hopkins, W.G., 2000. Measures of Reliability in Sports Medicine and Science. Sport. Med. 30, 375-381. https://doi.org/10.2165/00007256-200030050-00006

Jiménez-Reyes, P., Garcia-Ramos, A., Párraga-Montilla, J.A., Morcillo-Losa, J.A., Cuadrado-Peñafiel, V., Castaño-Zambudio, A., Samozino, P., Morin, J.-B., 2020. Seasonal Changes in the Sprint Acceleration Force-Velocity Profile of Elite Male Soccer Players. J. Strength Cond. Res. Publish Ah.

https://doi.org/10.1519/jsc.0000000000003513

Jiménez-Reyes, P., Samozino, P., García-Ramos, A., Cuadrado-Peñafiel, V., Brughelli, M., Morin, J.B., 2018. Relationship between vertical and horizontal force-velocity-power profiles in various sports and levels of practice. PeerJ 2018, e5937. https://doi.org/10.7717/peerj.5937

Mendiguchia, J., Edouard, P., Samozino, P., Brughelli, M., Cross, M., Ross, A., Gill, N., Morin, J.B., 2016. Field monitoring of sprinting power-force-velocity profile before, during and after hamstring injury: two case reports. J. Sports Sci. 34, 535-541. https://doi.org/10.1080/02640414.2015.1122207 
Mendiguchia, J., Samozino, P., Martinez-Ruiz, E., Brughelli, M., Schmikli, S., Morin, J.B., MendezVillanueva, A., 2014. Progression of mechanical properties during on-field sprint running after returning to sports from a hamstring muscle injury in soccer players. Int. J. Sports Med. 35, 690-695. https://doi.org/10.1055/s-0033-1363192

Morin, J.B., Petrakos, G., Jiménez-Reyes, P., Brown, S.R., Samozino, P., Cross, M.R., 2017. Very-heavy sled training for improving horizontal-force output in soccer players. Int. J. Sports Physiol. Perform. 12, 840-844. https://doi.org/10.1123/ijspp.2016-0444

Morin, J.B., Samozino, P., Murata, M., Cross, M.R., Nagahara, R., 2019. A simple method for computing sprint acceleration kinetics from running velocity data: Replication study with improved design. J. Biomech. 94, 82-87. https://doi.org/10.1016/j.jbiomech.2019.07.020

Nagahara, R., Matsubayashi, T., Matsuo, A., Zushi, K., 2014. Kinematics of transition during human accelerated sprinting. Biol. Open 3, 689-699. https://doi.org/10.1242/bio.20148284

Pinot, J., Grappe, F., 2015. A six-year monitoring case study of a top-10 cycling Grand Tour finisher. J. Sports Sci. 33, 907-914. https://doi.org/10.1080/02640414.2014.969296

Pinot, J., Grappe, F., 2011. The record power profile to assess performance in elite cyclists. Int. J. Sports Med. 32, 839-844. https://doi.org/10.1055/s-0031-1279773

Rabita, G., Dorel, S., Slawinski, J., Sàez-de-Villarreal, E., Couturier, A., Samozino, P., Morin, J.B., 2015. Sprint mechanics in world-class athletes: A new insight into the limits of human locomotion. Scand. J. Med. Sci. Sport. 25, 583-594. https://doi.org/10.1111/sms.12389

Samozino, P., Rabita, G., Dorel, S., Slawinski, J., Peyrot, N., Saez de Villarreal, E., Morin, J.B., 2016. A simple method for measuring power, force, velocity properties, and mechanical effectiveness in sprint running. Scand. J. Med. Sci. Sport. 26, 648-658. https://doi.org/10.1111/sms.12490 